\title{
SCALING AND SINGULAR LIMITS IN THE EQUATIONS OF CONTINUUM FLUID MECHANICS*
}

\author{
EDUARD FEIREISL ${ }^{\dagger}$
}

Key words. Navier-Stokes-Fourier system, dissipative solution, singular limit.

AMS subject classifications. 35Q30, 35Q35, 34E15.

1. Introduction. This is a survey on some recent results concerning scaling and the related singular limits in the models of complete fluids. We start by introducing the Navier-Stokes-Fourier system in the "entropy" form:

$$
\begin{gathered}
\partial_{t} \varrho+\operatorname{div}_{x}(\varrho \mathbf{u})=0, \\
\partial_{t}(\varrho \mathbf{u})+\operatorname{div}_{x}(\varrho \mathbf{u} \otimes \mathbf{u})+\nabla_{x} p(\varrho, \vartheta)=\operatorname{div}_{x} \mathbb{S}\left(\vartheta, \nabla_{x} \mathbf{u}\right), \\
\partial_{t}(\varrho s(\varrho, \vartheta))+\operatorname{div}_{x}(\varrho s(\varrho, \vartheta) \mathbf{u})+\operatorname{div}_{x}\left(\frac{\mathbf{q}\left(\vartheta, \nabla_{x} \vartheta\right)}{\vartheta}\right)=\sigma, \\
\sigma=\frac{1}{\vartheta}\left(\mathbb{S}: \nabla_{x} \mathbf{u}-\frac{\mathbf{q} \cdot \nabla_{x} \vartheta}{\vartheta}\right) .
\end{gathered}
$$

The system (1.1 - 1.3) governs the evolution of a compressible, viscous, and heat conducting fluid described in terms of the mass density $\varrho=\varrho(t, x)$, the absolute temperature $\vartheta=\vartheta(t, x)$, and the velocity field $\mathbf{u}=\mathbf{u}(t, x)$ in the Eulerian reference system, see Gallavotti [27]. Furthermore, the symbol $\mathbb{S}=\mathbb{S}\left(\vartheta, \nabla_{x} \mathbf{u}\right)$ stands for the viscous stress, here given by the standard Newton rheological law

$$
\mathbb{S}\left(\vartheta, \nabla_{x} \mathbf{u}\right)=\mu(\vartheta)\left(\nabla_{x} \mathbf{u}+\nabla_{x}^{t} \mathbf{u}-\frac{2}{3} \operatorname{div}_{x} \mathbf{u} \mathbb{I}\right)+\eta(\vartheta) \operatorname{div}_{x} \mathbf{u} \mathbb{I}
$$

and $\mathbf{q}\left(\vartheta, \nabla_{x} \vartheta\right)$ is the heat flux determined by the Fourier law

$$
\mathbf{q}=-\kappa(\vartheta) \nabla_{x} \vartheta
$$

Finally, $p=p(\varrho, \vartheta)$ is the pressure and $s=s(\varrho, \vartheta)$ the specific entropy related to the specific internal energy $e=e(\varrho, \vartheta)$ via Gibbs' equation

$$
\vartheta D s(\varrho, \vartheta)=D e(\varrho, \vartheta)+p(\varrho, \vartheta) D\left(\frac{1}{\varrho}\right) .
$$

In addition to (1.6) we impose the thermodynamic stability hypothesis

$$
\frac{\partial p(\varrho, \vartheta)}{\partial \varrho}>0, \frac{\partial e(\varrho, \vartheta)}{\partial \vartheta}>0
$$

\footnotetext{
*Received September 26, 2012; accepted for publication February 15, 2013.

$\dagger$ Institute of Mathematics of the Academy of Sciences of the Czech Republic, Žitná 25, 115 67 Praha 1, Czech Republic (feireisl@math.cas.cz); and Charles University in Prague, Faculty of Mathematics and Physics, Mathematical Institute, Sokolovská 83, 18675 Praha 8, Czech Republic. The author acknowledges the support of the project LL1202 in the programme ERC-CZ funded by the Ministry of Education, Youth and Sports of the Czech Republic.
} 
that will play a crucial role in the analysis (see Callen [10] for the physical background of (1.6), (1.7)).

Equations (1.1-1.3) represent our primitive system that is supposed to provide a complete description of a given fluid in motion. Given the enormous scope of applications of continuum fluid mechanics, solutions of the Navier-Stokes-Fourier system describe the motion of general gases and compressible liquids around or without presence of rigid bodies, the atmosphere and oceans in meteorology, and even the evolution of gaseous stars. Obviously, these phenomena may occur on very different time and spatial scales, where simplified models may provide equally good if not better picture of reality. Our goal is to show how these models can be rigorously derived as singular limits of a scaled version of (1.1-1.3), where certain characteristic numbers tend to zero or become excessively large.

1.1. Scaling and dimensionless equations. The method of scaling is well known and frequently used in engineering. Instead of considering the physical quantities in their original (typically S.I.) units, we replace a quantity $X$ by $X / X_{\text {char }}$, where $X_{\text {char }}$ is the characteristic value of $X$. Applying this procedure to the system (1.11.3) and keeping the same symbols for physical quantities and their dimensionless counterparts, we arrive at the following scaled Navier-Stokes-Fourier system:

$$
\begin{gathered}
{[\operatorname{Sr}] \partial_{t} \varrho+\operatorname{div}_{x}(\varrho \mathbf{u})=0} \\
{[\operatorname{Sr}] \partial_{t}(\varrho \mathbf{u})+\operatorname{div}_{x}(\varrho \mathbf{u} \otimes \mathbf{u})+\left[\frac{1}{\mathrm{Ma}^{2}}\right] \nabla_{x} p(\varrho, \vartheta)=\left[\frac{1}{\operatorname{Re}}\right] \operatorname{div}_{x} \mathbb{S}\left(\vartheta, \nabla_{x} \mathbf{u}\right),} \\
{[\operatorname{Sr}] \partial_{t}(\varrho s(\varrho, \vartheta))+\operatorname{div}_{x}(\varrho s(\varrho, \vartheta) \mathbf{u})+\left[\frac{1}{\mathrm{Pe}}\right] \operatorname{div}_{x}\left(\frac{\mathbf{q}}{\vartheta}\right)=\sigma} \\
\sigma=\frac{1}{\vartheta}\left(\left[\frac{\mathrm{Ma}^{2}}{\mathrm{Re}}\right] \mathbb{S}: \nabla_{x} \mathbf{u}-\left[\frac{1}{\mathrm{Pe}}\right] \frac{\mathbf{q} \cdot \nabla_{x} \vartheta}{\vartheta}\right)
\end{gathered}
$$

with the characteristic numbers:

- Strouhal number (Čeněk Strouhal [1850-1922]):

$$
[\mathrm{Sr}]=\frac{\text { length }_{\text {char }}}{\text { time }_{\text {char velocity }} \text { char }} ;
$$

- Mach number (Ernst Mach [1838-1916]):

$$
[\mathrm{Ma}]=\frac{\text { velocity }_{\text {char }}}{\sqrt{\text { pressure }_{\text {char }} / \text { density }_{\text {char }}}} ;
$$

- Reynolds number (Osborne Reynolds [1842-1912]):

$$
[\mathrm{Re}]=\frac{\text { density }_{\text {char }} \text { velocity }_{\text {char }} \text { length }_{\text {char }}}{\text { viscosity }_{\text {char }}} ;
$$

- Péclet number (Jean Claude Eugène Péclet [1793-1857]):

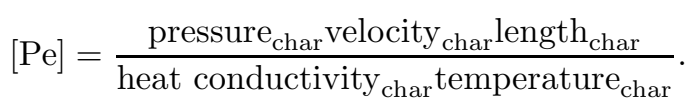


As a matter of fact, specific values of characteristic numbers may correspond to physically different systems. For instance, high Reynolds number may be associated to low viscosity of the fluid or to extremely large length scales. We refer to the survey of Klein et al [37] for a thorough discussion of singular limits and the applications of scaling in numerical analysis.

1.2. Inviscid, incompressible limit. We focus on the situation when

$$
\mathrm{Sr}=1, \mathrm{Ma}=\varepsilon, \operatorname{Re}=\varepsilon^{-a}, \mathrm{Pe}=\varepsilon^{-b}, a, b>0,
$$

where $\varepsilon>0$ is a small parameter. Our goal is to identify the limit system for $\varepsilon \rightarrow 0$, meaning the inviscid, incompressible limit of the scaled Navier-Stokes-Fourier system:

$$
\begin{gathered}
\partial_{t} \varrho+\operatorname{div}_{x}(\varrho \mathbf{u})=0, \\
\partial_{t}(\varrho \mathbf{u})+\operatorname{div}_{x}(\varrho \mathbf{u} \otimes \mathbf{u})+\frac{1}{\varepsilon^{2}} \nabla_{x} p(\varrho, \vartheta)=\varepsilon^{a} \operatorname{div}_{x} \mathbb{S}\left(\vartheta, \nabla_{x} \mathbf{u}\right), \\
\partial_{t}(\varrho s(\varrho, \vartheta))+\operatorname{div}_{x}(\varrho s(\varrho, \vartheta) \mathbf{u})+\varepsilon^{b} \operatorname{div}_{x}\left(\frac{\mathbf{q}}{\vartheta}\right)=\sigma, \\
\sigma=\frac{1}{\vartheta}\left(\varepsilon^{2+a} \mathbb{S}: \nabla_{x} \mathbf{u}-\varepsilon^{b} \frac{\mathbf{q} \cdot \nabla_{x} \vartheta}{\vartheta}\right),
\end{gathered}
$$

supplemented with the initial conditions:

$$
\varrho(0, \cdot)=\varrho_{0, \varepsilon}=\bar{\varrho}+\varepsilon \varrho_{0, \varepsilon}^{(1)}, \vartheta(0, \cdot)=\vartheta_{0, \varepsilon}=\bar{\vartheta}+\varepsilon \vartheta_{0, \varepsilon}^{(1)}, \mathbf{u}(0, \cdot)=\mathbf{u}_{0, \varepsilon},
$$

where the reference values $\bar{\varrho}, \bar{\vartheta}$ are positive constants. Note that the initial distribution of the density and the temperature are prepared anticipating the constant values expected in the asymptotic limit for $\varepsilon \rightarrow 0$.

1.3. Limit system. Formally, it is easy to identify the limit system of equations. Indeed the fact that the Mach number is small indicates incompressibility of the limit fluid flow; whence the limit system reads:

$$
\begin{gathered}
\operatorname{div}_{x} \mathbf{v}=0, \\
\partial_{t} \mathbf{v}+\operatorname{div}_{x}(\mathbf{v} \otimes \mathbf{v})+\nabla_{x} \Pi=0, \\
\partial_{t} \mathcal{T}+\mathbf{v} \cdot \nabla_{x} \mathcal{T}=0,
\end{gathered}
$$

which is nothing other than the incompressible Euler system, supplemented with the transport equation for the temperature deviation $\mathcal{T}$,

$$
\mathcal{T} \approx \lim _{\varepsilon \rightarrow 0} \frac{\vartheta_{\varepsilon}-\bar{\vartheta}}{\varepsilon}
$$


1.4. Boundary conditions. Real fluid systems are typically confined to a physical space - a domain $\Omega \subset R^{3}$. Accordingly, the boundary behavior of certain quantities must be specified. In order to avoid the so far unsurmountable problem of the boundary layer in the inviscid limit, see for instance Kato [32], we restrict ourselves to the Navier slip boundary condition

$$
\left.\mathbf{u} \cdot \mathbf{n}\right|_{\partial \Omega}=0, \varepsilon^{c}\left[\mathbb{S}\left(\vartheta, \nabla_{x} \mathbf{u}\right) \mathbf{n}\right]_{\tan }+\left.\beta(\vartheta) \mathbf{u}\right|_{\partial \Omega}=0, c, \beta>0 .
$$

In addition, we impose the no-flux condition for the total energy, specifically, in terms of the heat flux $\mathbf{q}$,

$$
\left.\mathbf{q}\left(\vartheta, \nabla_{x} \vartheta\right) \cdot \mathbf{n}\right|_{\partial \Omega}=-\left.\beta \varepsilon^{d}|\mathbf{u}|^{2}\right|_{\partial \Omega}, d=2+a-c-b .
$$

Condition (1.19) implies, in particular, that the total energy of the system is a conserved quantity:

$$
\frac{\mathrm{d}}{\mathrm{d} t} \int_{\Omega}\left(\varepsilon^{2} \varrho|\mathbf{u}|^{2}+\varrho e(\varrho, \vartheta)\right) \mathrm{d} x=0 .
$$

1.5. Singular limit. Our main goal is to discuss the singular limit process from the scaled Navier-Stokes-Fourier system $(1.11$ - 1.14), supplemented with the boundary conditions $(1.18),(1.19)$, to the target Euler system $(1.15-1.17)$ as $\varepsilon \rightarrow 0$. Our working plan reads as follows:

- In Section 2, we introduce the relative entropy inequality together with the concept of dissipative solutions to the (primitive) Navier-Stokes-Fourier system.

- We use the relative entropy inequality to derive stability estimates for the solutions of the scaled system, see Section 3.

- In Section 4, we analyze the asymptotic behavior of acoustic waves and show the relevant dispersive estimates.

- Section 5 contains final comments and concluding remarks.

2. Weak and dissipative solutions. Solutions of the system (1.11 - 1.13), (1.18), (1.19) satisfy, together with the total energy balance (1.20), the total entropy production relation in the form

$$
\frac{\mathrm{d}}{\mathrm{d} t} \int_{\Omega} \varrho s(\varrho, \vartheta) \mathrm{d} x=\int_{\Omega} \sigma \mathrm{d} x+\varepsilon^{2+a-c} \int_{\partial \Omega} \frac{\beta}{\vartheta}|\mathbf{u}|^{2} \mathrm{~d} \mathrm{~S}_{x} .
$$

Thus, adding (1.20), (2.1) together, we obtain

$$
\begin{aligned}
\frac{\mathrm{d}}{\mathrm{d} t} \int_{\Omega}\left[\frac{1}{2} \varrho|\mathbf{u}|^{2}\right. & \left.+\frac{1}{\varepsilon^{2}}(\varrho e(\varrho, \vartheta)-\Theta \varrho s(\varrho, \vartheta))\right] \mathrm{d} x \\
+ & \frac{\Theta}{\varepsilon^{2}} \int_{\Omega} \sigma \mathrm{d} x+\Theta \varepsilon^{a-c} \int_{\partial \Omega} \frac{\beta}{\vartheta}|\mathbf{u}|^{2} \mathrm{~d} \mathrm{~S}_{x}=0
\end{aligned}
$$

for any positive constant $\Theta$. Relation (2.2) is usually termed total dissipation balance. The functional

$$
(\varrho, \vartheta, \mathbf{u}) \mapsto \int_{\Omega}\left[\frac{1}{2} \varrho|\mathbf{u}|^{2}+\frac{1}{\varepsilon^{2}}(\varrho e(\varrho, \vartheta)-\Theta \varrho s(\varrho, \vartheta))\right] \mathrm{d} x
$$

turns out to be a Lyapunov function for the Navier-Stokes-Fourier system. 
2.1. Ballistic free energy. The functional

$$
H_{\Theta}(\varrho, \vartheta)=\varrho(e(\varrho, \vartheta)-\Theta s(\varrho, \vartheta))
$$

is called ballistic free energy, see Ericksen [19].

It is a routine matter to compute

$$
\frac{\partial^{2} H_{\Theta}(\varrho, \Theta)}{\partial \varrho^{2}}=\frac{1}{\varrho} \frac{\partial p(\varrho, \Theta)}{\partial \varrho} \text { and } \frac{\partial H_{\Theta}(\varrho, \theta)}{\partial \vartheta}=\varrho \frac{\partial s(\varrho, \vartheta)}{\partial \vartheta}(\vartheta-\Theta) \text {. }
$$

Using the hypothesis of thermodynamic stability (1.7) we therefore conclude that

$$
\varrho \mapsto H_{\Theta}(\varrho, \Theta) \text { is strictly convex, }
$$

and

$$
\vartheta \mapsto H_{\Theta}(\varrho, \vartheta) \text { is decreasing for } \vartheta<\Theta \text { and increasing for } \vartheta>\Theta \text { for any fixed } \varrho \text {. }
$$

2.2. Relative entropy. Motivated by the discussion in the preceding section, we introduce the relative entropy functional in the form

$$
\begin{aligned}
& \mathcal{E}(\varrho, \vartheta, \mathbf{u} \mid r, \Theta, \mathbf{U}) \\
& =\int_{\Omega}\left(\frac{1}{2} \varrho|\mathbf{u}-\mathbf{U}|^{2}+H_{\Theta}(\varrho, \vartheta)-\frac{\partial H_{\Theta}(r, \Theta)}{\partial \varrho}(\varrho-r)-H_{\Theta}(r, \Theta)\right) \mathrm{d} x .
\end{aligned}
$$

In the light of the coercivity properties $(2.4),(2.5)$, it is easy to check that the relative entropy represents a kind of distance between the trio $(\varrho, \vartheta, \mathbf{u})$ and $(r, \Theta, \mathbf{U})$. Going back to the total dissipation inequality (2.2) we obtain

$$
\frac{\mathrm{d}}{\mathrm{d} t} \mathcal{E}_{\varepsilon}(\varrho, \vartheta, \mathbf{u} \mid \bar{\varrho}, \bar{\vartheta}, 0)+\frac{\bar{\vartheta}}{\varepsilon^{2}} \int_{\Omega} \sigma \mathrm{d} x+\bar{\vartheta} \varepsilon^{a-c} \int_{\partial \Omega} \frac{\beta}{\vartheta}|\mathbf{u}|^{2} \mathrm{~d} \mathrm{~S}_{x}=0
$$

where we have set

$$
\begin{aligned}
& \mathcal{E}_{\varepsilon}(\varrho, \vartheta, \mathbf{u} \mid r, \Theta, \mathbf{U}) \\
& =\int_{\Omega}\left[\frac{1}{2} \varrho|\mathbf{u}-\mathbf{U}|^{2}+\frac{1}{\varepsilon^{2}}\left(H_{\Theta}(\varrho, \vartheta)-\frac{\partial H_{\Theta}(r, \Theta)}{\partial \varrho}(\varrho-r)-H_{\Theta}(r, \Theta)\right)\right] \mathrm{d} x,
\end{aligned}
$$

and where $\bar{\varrho}, \bar{\vartheta}$ are the positive constants appearing in the initial conditions (1.14), chosen in such a way that, at least formally,

$$
\int_{\Omega}(\varrho-\bar{\varrho}) \mathrm{d} x=0
$$

If $\Omega$ is a bounded domain, the satisfaction of (2.9) is guaranteed if the perturbation $\varrho_{0, \varepsilon}^{(1)}$ is taken of zero integral mean as the total mass of the fluid

$$
M_{0}=\int_{\Omega} \varrho(t, \cdot) \mathrm{d} x
$$


is a constant of motion. In general, the constants $\bar{\varrho}, \bar{\vartheta}$ will be always chosen in such a way that $(2.7)$ holds. The trio $(\bar{\varrho}, \bar{\vartheta}, 0)$ is trivially a solution to the Navier-StokesFourier system $(1.1-1.3)$ that is called a static state. In view of the coercivity properties of the relative entropy established in (2.4), (2.5), relation (2.8) yields stability of the "static" states with respect to perturbations.

Our next goal is to derive a relation (inequality) similar to $(2.7)$ provided $(\varrho, \vartheta, \mathbf{u})$ is a weak solution of the Navier-Stokes-Fourier system, and $(r, \Theta, \mathbf{U})$ is an arbitrary trio of "test functions" satisfying natural boundary conditions. To this end, a short excursion in the theory of weak solutions to the Navier-Stokes-Fourier system is needed.

2.3. Weak solutions. Following [21, Chapter 3] we introduce the concept of weak solution to the Navier-Stokes-Fourier system (1.1 - 1.3), with the boundary conditions (1.18), (1.19), and the initial conditions

$$
\varrho(0, \cdot)=\varrho_{0}, \vartheta(0, \cdot)=\vartheta_{0}, \mathbf{u}(0, \cdot)=\mathbf{u}_{0} .
$$

To simplify presentation, we suppose that $\Omega \subset R^{3}$ is a bounded domain with smooth boundary.

2.3.1. Constitutive relations. Besides the existing restrictions imposed on the thermodynamic functions $p, e$, and $s$ through Gibbs' equation (1.6) and the thermodynamic stability hypothesis (1.7), we introduce rather technical but still physically grounded assumptions required by the existence theory developed in [21]. More specifically, we suppose that the pressure $p$ is given in the form

$$
p(\varrho, \vartheta)=\vartheta^{5 / 2} P\left(\frac{\varrho}{\vartheta^{3 / 2}}\right)+\frac{a}{3} \vartheta^{4}, a>0, P(0)=0 .
$$

Here, the term proportional to $\vartheta^{4}$ is attributed to the radiation pressure, while the specific form

$$
\vartheta^{5 / 2} P\left(\frac{\varrho}{\vartheta^{3 / 2}}\right)
$$

can be derived from the Gibbs' equation (1.6) as the universal formula for the monoatomic gas satisfying

$$
p(\varrho, \vartheta)=\frac{2}{3} \varrho e(\varrho, \vartheta)
$$

see [21, Chapter 1].

Accordingly, we take

$$
e(\varrho, \vartheta)=\frac{3}{2} \vartheta\left(\frac{\vartheta^{3 / 2}}{\varrho}\right) P\left(\frac{\varrho}{\vartheta^{3 / 2}}\right)+\frac{a}{\varrho} \vartheta^{4},
$$

and

$$
s(\varrho, \vartheta)=S\left(\frac{\varrho}{\vartheta^{3 / 2}}\right)+\frac{4 a}{3} \frac{\vartheta^{3}}{\varrho},
$$

where

$$
S^{\prime}(Z)=-\frac{3}{2} \frac{\frac{5}{3} P(Z)-P^{\prime}(Z) Z}{Z^{2}} .
$$


The thermodynamic stability hypothesis (1.7) stated in terms of the structural properties of the function $P$ gives rise to:

$$
P^{\prime}(Z)>0,0<\frac{\frac{5}{3} P(Z)-P^{\prime}(Z) Z}{Z^{2}}<c \text { for all } Z>0 .
$$

In particular, the function $Z \mapsto P(Z) / Z^{5 / 3}$ is non-increasing, and we take

$$
\lim _{Z \rightarrow \infty} \frac{P(Z)}{Z^{5 / 3}}=p_{\infty}>0
$$

Finally, the Third law of thermodynamics is imposed through

$$
\lim _{Z \rightarrow \infty} S(Z)=0 .
$$

As for the transport coefficients $\mu, \lambda, \beta$ and $\kappa$, we shall assume that they are continuously differentiable functions of the absolute temperature $\vartheta \in[0, \infty)$ satisfying:

$$
\mu \in C^{1}[0, \infty) \text { is globally Lipschitz continuous, } 0<\underline{\mu}(1+\vartheta) \leq \mu(\vartheta),
$$

$$
0 \leq \eta(\vartheta) \leq \bar{\eta}(1+\vartheta)
$$

and

$$
\underline{\beta}(1+\vartheta) \leq \beta(\vartheta) \leq \bar{\beta}(1+\vartheta), \underline{\kappa}\left(1+\vartheta^{3}\right) \leq \kappa(\vartheta) \leq \bar{\kappa}\left(1+\vartheta^{3}\right) .
$$

2.3.2. Variational formulation. We introduce a weak (variational) formulation of the Navier-Stokes-Fourier system, taking into account the boundary conditions (1.18), (1.19), together with the initial conditions (2.10), see [21, Chapter 3].

We say that a trio $(\varrho, \vartheta, \mathbf{u})$ is a weak solution of the (unscaled) Navier-StokesFourier system $(1.1-1.3)$ if

$$
\begin{gathered}
\varrho \geq 0, \varrho \in C_{\text {weak }}\left([0, T] ; L^{5 / 3}(\Omega)\right) \cap L^{q}((0, T) \times \Omega) \text { for a certain } q>\frac{5}{3}, \\
\vartheta>0 \text { a.a. in }(0, T) \times \Omega, \vartheta \in L^{\infty}\left(0, T ; L^{4}(\Omega)\right) \cap L^{2}\left(0, T ; W^{1,2}(\Omega)\right), \\
\qquad \log (\vartheta) \in L^{2}\left(0, T ; W^{1,2}(\Omega)\right), \\
\mathbf{u} \in L^{2}\left(0, T ; W^{1,2}\left(\Omega ; R^{3}\right)\right),\left.\mathbf{u} \cdot \mathbf{n}\right|_{\partial \Omega}=0, \varrho \mathbf{u} \in C_{\text {weak }}\left([0, T] ; L^{5 / 4}(\Omega)\right),
\end{gathered}
$$

and the following integral identities are satisfied:

$$
\left[\int_{\Omega} \varrho \varphi(t, \cdot) \mathrm{d} x\right]_{t=0}^{\tau}=\int_{0}^{\tau} \int_{\Omega}\left(\varrho \partial_{t} \varphi+\varrho \mathbf{u} \cdot \nabla_{x} \varphi\right) \mathrm{d} x \mathrm{~d} t
$$

for any $\tau \in[0, T]$, and any $\varphi \in C_{c}^{\infty}([0, T] \times \bar{\Omega})$;

$$
\begin{aligned}
& \quad\left[\int_{\Omega} \varrho \mathbf{u} \cdot \varphi(t, \cdot) \mathrm{d} x\right]_{t=0}^{\tau} \\
& =\int_{0}^{\tau} \int_{\Omega}\left(\varrho \mathbf{u} \cdot \partial_{t} \varphi+(\varrho \mathbf{u} \times \mathbf{u}): \nabla_{x} \varphi+p(\varrho, \vartheta) \operatorname{div}_{x} \varphi-\mathbb{S}: \nabla_{x} \varphi\right) \mathrm{d} x \mathrm{~d} t \\
& \quad-\int_{0}^{\tau} \int_{\partial \Omega} \beta \mathbf{u} \cdot \varphi \mathrm{dS}_{x}
\end{aligned}
$$


for any $\tau \in[0, T]$, and any $\varphi \in C_{c}^{\infty}\left([0, T] \times \bar{\Omega} ; R^{3}\right),\left.\varphi \cdot \mathbf{n}\right|_{\partial \Omega}=0$;

$$
\begin{aligned}
& {\left[\int_{\Omega} \varrho s(\varrho, \vartheta) \varphi(t, \cdot) \mathrm{d} x\right]_{t=0}^{\tau}} \\
& \geq \int_{0}^{\tau} \int_{\Omega}\left(\varrho s(\varrho, \vartheta) \partial_{t} \varphi+\varrho s(\varrho, \vartheta) \mathbf{u} \cdot \nabla_{x} \varphi+\frac{\mathbf{q}}{\vartheta} \cdot \nabla_{x} \varphi-\mathbb{S}\right) \mathrm{d} x \mathrm{~d} t \\
& \quad+\int_{0}^{\tau} \int_{\Omega} \frac{1}{\vartheta}\left(\mathbb{S}: \nabla_{x} \mathbf{u}-\frac{\mathbf{q} \cdot \nabla_{x} \vartheta}{\vartheta}\right) \varphi \mathrm{d} x \mathrm{~d} t+\int_{0}^{\tau} \int_{\partial \Omega} \beta|\mathbf{u}|^{2} \varphi \mathrm{d} \mathrm{S}_{x} \mathrm{~d} t
\end{aligned}
$$

for a.a. $\tau \in[0, T]$, and any $\varphi \in C_{c}^{\infty}([0, T] \times \bar{\Omega}), \varphi \geq 0$.

Since the weak formulation is stated for the unscaled system, we have taken $\varepsilon=1$ in the boundary conditions (1.18), (1.19). Note that the initial conditions are "hidden" in the quantities on the left-hand side of the above integral formulas.

While the integral identities (2.23), (2.24) represent the standard weak formulation of the equations $(1.1),(1.2)$, the reader will have noticed that the entropy balance (1.3) has been replaced by inequality $(2.25)$ corresponding to the entropy production rate

$$
\sigma \geq \frac{1}{\vartheta}\left(\mathbb{S}: \nabla_{x} \mathbf{u}-\frac{\mathbf{q} \cdot \nabla_{x} \vartheta}{\vartheta}\right)
$$

In order to compensate for this obvious lack of information, the variational formulation will be augmented, similarly to [21, Chapter 3], by the total energy balance

$$
\left[\int_{\Omega}\left(\frac{1}{2} \varrho|\mathbf{u}|^{2}+\varrho e(\varrho, \vartheta)\right) \mathrm{d} x\right]_{t=0}^{\tau}=0 \text { for a.a. } \tau \in[0, T] .
$$

It can be shown that any weak solution that is sufficiently smooth solves the system of equations $(1.1-1.3)$, see [21, Chapter 2]. The resulting concept of weak solution is mathematically tractable. In particular, we report the following global-intime existence result in the class of weak solutions, see [21, Theorems 3.1,3.2].

TheOREM 2.1. Let $\Omega \subset R^{3}$ be a bounded domain of class $C^{2+\nu}$. Suppose that the thermodynamic functions $p, e, s$, and the transport coefficients $\mu, \eta, \beta, \kappa$ comply with the structural restrictions introduced in Section 2.3.1. Finally, let the initial data be taken such that

$$
\varrho_{0}>0, \vartheta_{0}>0 \text { a.a. in } \Omega, E_{0}=\int_{\Omega}\left(\frac{1}{2} \varrho_{0}\left|\mathbf{u}_{0}\right|^{2}+\varrho_{0} e\left(\varrho_{0}, \vartheta_{0}\right)\right) \mathrm{d} x<\infty .
$$

Then the Navier-Stokes-Fourier possesses a weak solution in $(0, T) \times \Omega$ for any $T>0$ in the sense specified through (2.20 - 2.26).

Possible generalizations with respect to the structural properties of $p, e$, and $s$ as well as relaxation of the growth conditions $(2.17),(2.18)$ are discussed at length in $\left[21\right.$, Chapter 3]. We also remark that the initial density $\varrho_{0}$ may be taken only nonnegative in $\Omega$, however, such a generalization seems to be at odds with the standard derivation of the Navier-Stokes system as a model of non-dilute fluids.

An alternative approach in the framework of weak solutions to the Navier-StokesFourier system was proposed by Bresch and Desjardins [6], [7]. They assume that the viscosity coefficients depend on the density $\varrho$ in a special way and derive a priori bounds on the density gradient in certain function spaces. 
2.4. Dissipative solutions. The dissipative solutions of the Navier-StokesFourier system will be characterized by relative entropy inequality we are going to derive. After a bit tedious but absolutely routine manipulation we obtain

$$
\begin{aligned}
& {[\mathcal{E}(\varrho, \vartheta, \mathbf{u} \mid r, \Theta, \mathbf{U})]_{t=0}^{\tau}} \\
& +\int_{0}^{\tau} \int_{\Omega} \frac{\Theta}{\vartheta}\left(\mathbb{S}\left(\vartheta, \nabla_{x} \mathbf{u}\right): \nabla_{x} \mathbf{u}-\frac{\mathbf{q}\left(\vartheta, \nabla_{x} \vartheta\right) \cdot \nabla_{x} \vartheta}{\vartheta}\right) \mathrm{d} x \mathrm{~d} t+\int_{0}^{\tau} \int_{\partial \Omega} \frac{\Theta \beta}{\vartheta}|\mathbf{u}|^{2} \mathrm{~d} \mathbf{S}_{x} \mathrm{~d} t \\
& \leq \int_{0}^{\tau} \int_{\Omega}\left(\varrho\left(\partial_{t} \mathbf{U}+\mathbf{u} \cdot \nabla_{x} \mathbf{U}\right) \cdot(\mathbf{U}-\mathbf{u})+\mathbb{S}\left(\vartheta, \nabla_{x} \mathbf{u}\right): \nabla_{x} \mathbf{U}\right) \mathrm{d} x \mathrm{~d} t d t \\
& +\int_{0}^{\tau} \int_{\partial \Omega} \beta \mathbf{u} \cdot \mathbf{U} \mathrm{d} \mathbf{S}_{x}+\int_{0}^{\tau} \int_{\Omega}\left[(p(r, \Theta)-p(\varrho, \vartheta)) \mathrm{div} \mathbf{U}+\frac{\varrho}{r}(\mathbf{U}-\mathbf{u}) \cdot \nabla_{x} p(r, \Theta)\right] \mathrm{d} x \mathrm{~d} t \\
& -\int_{0}^{\tau} \int_{\Omega}\left(\varrho(s(\varrho, \vartheta)-s(r, \Theta)) \partial_{t} \Theta+\varrho(s(\varrho, \vartheta)-s(r, \Theta)) \mathbf{u} \cdot \nabla_{x} \Theta+\frac{\mathbf{q}\left(\vartheta, \nabla_{x} \vartheta\right)}{\vartheta} \cdot \nabla_{x} \Theta\right) \mathrm{d} x \mathrm{~d} t \\
& +\int_{0}^{\tau} \int_{\Omega} \frac{r-\varrho}{r}\left(\partial_{t} p(r, \Theta)+\mathbf{U} \cdot \nabla_{x} p(r, \Theta)\right) \mathrm{d} x \mathrm{~d} t
\end{aligned}
$$

for any (smooth) solution $(\varrho, \vartheta, \mathbf{u})$ of the Navier-Stokes-Fourier system and any trio of smooth "test" functions $(r, \Theta, \mathbf{U})$ satisfying

$$
r>0, \Theta>0,\left.\mathbf{U} \cdot \mathbf{n}\right|_{\partial \Omega}=0,
$$

see [23].

Relation (2.27) is called relative entropy inequality. Our next observation is that it can be extended to the class of weak solutions. Indeed we may write

$$
\mathcal{E}(\varrho, \vartheta, \mathbf{u} \mid r, \Theta, \mathbf{U})=\sum_{i=1}^{6} I_{i},
$$

where

$$
\begin{aligned}
& I_{1}=\int_{\Omega}\left(\frac{1}{2} \varrho|\mathbf{u}|^{2}+\varrho e(\varrho, \vartheta)\right) \mathrm{d} x, \\
& I_{2}=-\int_{\Omega} \varrho \mathbf{u} \cdot \mathbf{U} \mathrm{d} x, \\
& I_{3}=\int_{\Omega} \frac{1}{2} \varrho|\mathbf{U}|^{2} \mathrm{~d} x, \\
& I_{4}=-\int_{\Omega} \varrho s(\varrho, \vartheta) \Theta \mathrm{d} x, \\
& I_{5}=-\int_{\Omega} \frac{\partial H_{\Theta}(r, \Theta)}{\partial \varrho} \varrho \mathrm{d} x,
\end{aligned}
$$

and

$$
I_{6}=\int_{\Omega}\left(\frac{\partial H_{\Theta}(r, \Theta)}{\partial \varrho} r-H(r, \Theta)\right) \mathrm{d} x .
$$

Since the functions $(r, \Theta, \mathbf{U})$ are smooth and $\mathbf{U}$ satisfies the relevant boundary conditions, all quantities $\left[I_{i}\right]_{t=0}^{\tau}$ can be expressed by means of the weak formulation (2.23 - 2.26), cf. [23] for details. 
Motivated by a similar definition introduced by DiPerna and Lions [41] in the context of inviscid fluids, we say that $(\varrho, \vartheta, \mathbf{u})$ is a dissipative solution to the NavierStokes-Fourier system if the relative entropy inequality (2.27) holds for all smooth test functions satisfying (2.28).

As we have just observed, the weak solutions of the Navier-Stokes-Fourier system in a bounded regular domain $\Omega$ are dissipative solutions. The relative entropy inequality is a powerful tool that has been successfully applied to the following topics:

- the unconditional stability of the static states and attractors for the full Navier-Stokes-Fourier system, see [25];

- the problem of weak-strong uniqueness, see [23];

- the singular limits for low Mach and high Reynolds and Péclet numbers, see $[22]$.

Here, we focus on the last issue discussing the limit $\varepsilon \rightarrow 0$ in the scaled system.

2.4.1. Possible extensions. The concept of dissipative solution can be easily extended to problems on general unbounded domains. In such a situation, the constants $\bar{\varrho}, \bar{\vartheta}$ are taken to characterize the far field behavior, specifically,

$$
\varrho \rightarrow \bar{\varrho}, \vartheta \rightarrow \bar{\vartheta} \text { as }|x| \rightarrow \infty
$$

Moreover, we shall always assume that the velocity vanishes for large $x$,

$$
\mathbf{u} \rightarrow 0 \text { as }|x| \rightarrow \infty
$$

Now, the relative entropy inequality remains formally the same as (2.27), where, in addition to (2.28), the test functions $r, \Theta$, $\mathbf{U}$ admit suitable "far field" behavior. We may assume that

$$
r-\bar{\varrho}, \vartheta-\bar{\vartheta}, \mathbf{U} \in C_{c}^{\infty}([0, T] \times \bar{\Omega})
$$

or that they decay rapidly to their asymptotic limits depending on the integrability of the weak solutions.

As the relative entropy inequality contains a complete piece of information we need to perform the singular limit we are interested in, we focus in the future only on dissipative solutions. Note that the global-in-time existence of dissipative solutions occupying a general unbounded physical space can be easily shown via the method of invading domains, where we construct weak (dissipative) solutions on a family of bounded domains

$$
\Omega_{R}=\Omega \cap\{|x|<R\}
$$

and let $R \rightarrow \infty$, see Jesslé, Jin, and Novotný [29].

Since in the future we will deal exclusively with the scaled system $(1.11-1.13)$, 
we start by reformulating $(2.27)$ in the $\varepsilon$-framework:

$$
\begin{aligned}
& {\left[\mathcal{E}_{\varepsilon}(\varrho, \vartheta, \mathbf{u} \mid r, \Theta, \mathbf{U})\right]_{t=0}^{\tau}} \\
& +\int_{0}^{\tau} \int_{\Omega} \frac{\Theta}{\vartheta}\left(\varepsilon^{a} \mathbb{S}\left(\vartheta, \nabla_{x} \mathbf{u}\right): \nabla_{x} \mathbf{u}-\varepsilon^{b-2} \frac{\mathbf{q}\left(\vartheta, \nabla_{x} \vartheta\right) \cdot \nabla_{x} \vartheta}{\vartheta}\right) \mathrm{d} x \mathrm{~d} t+\varepsilon^{a-c} \int_{0}^{\tau} \int_{\partial \Omega} \frac{\Theta \beta}{\vartheta}|\mathbf{u}|^{2} \mathrm{~d} \mathrm{~S}_{x} \mathrm{~d} t \\
& \leq \int_{0}^{\tau} \int_{\Omega}\left(\varrho\left(\partial_{t} \mathbf{U}+\mathbf{u} \cdot \nabla_{x} \mathbf{U}\right) \cdot(\mathbf{U}-\mathbf{u})+\varepsilon^{a} \mathbb{S}\left(\vartheta, \nabla_{x} \mathbf{u}\right): \nabla_{x} \mathbf{U}\right) \mathrm{d} x \mathrm{~d} t+\varepsilon^{a-c} \int_{0}^{\tau} \int_{\partial \Omega} \beta \mathbf{u} \cdot \mathbf{U} \mathrm{d} \mathbf{S}_{x} \mathrm{~d} t \\
& +\frac{1}{\varepsilon^{2}} \int_{0}^{\tau} \int_{\Omega}\left[(p(r, \Theta)-p(\varrho, \vartheta)) \operatorname{div} \mathbf{U}+\frac{\varrho}{r}(\mathbf{U}-\mathbf{u}) \cdot \nabla_{x} p(r, \Theta)\right] \mathrm{d} x \mathrm{~d} t \\
& -\frac{1}{\varepsilon^{2}} \int_{0}^{\tau} \int_{\Omega}\left(\varrho(s(\varrho, \vartheta)-s(r, \Theta)) \partial_{t} \Theta+\varrho(s(\varrho, \vartheta)-s(r, \Theta)) \mathbf{u} \cdot \nabla_{x} \Theta+\varepsilon^{b} \frac{\mathbf{q}\left(\vartheta, \nabla_{x} \vartheta\right)}{\vartheta} \cdot \nabla_{x} \Theta\right) \mathrm{d} x \mathrm{~d} t \\
& +\frac{1}{\varepsilon^{2}} \int_{0}^{\tau} \int_{\Omega} \frac{r-\varrho}{r}\left(\partial_{t} p(r, \Theta)+\mathbf{U} \cdot \nabla_{x} p(r, \Theta)\right) \mathrm{d} x \mathrm{~d} t
\end{aligned}
$$

for all test functions

$$
\begin{aligned}
& r>0, \Theta>0,\left.\mathbf{U} \cdot \mathbf{n}\right|_{\partial \Omega} \\
& =0, \varrho-r, \vartheta-\Theta \in C_{c}^{\infty}([0, T] \times \bar{\Omega}), \mathbf{U} \in C_{c}^{\infty}\left([0, T] \times \bar{\Omega} ; R^{3}\right) .
\end{aligned}
$$

As we shall see in the next section, the integrability properties of the dissipative solutions on unbounded domains are slightly different from those on bounded ones. As a matter of fact, they follow directly from (2.32).

3. Uniform bounds, stability. Anticipating the existence of global-in-time dissipative solutions $\left(\varrho_{\varepsilon}, \vartheta_{\varepsilon}, \mathbf{u}_{\varepsilon}\right)$ satisfying the relative entropy inequality (2.32), we derive uniform bounds independent of $\varepsilon \rightarrow 0$. To this end, it is convenient to introduce the following notation:

$$
h=h_{\mathrm{ess}}+h_{\mathrm{res}}, h_{\mathrm{ess}}=\Psi\left(\varrho_{\varepsilon}, \vartheta_{\varepsilon}\right) h, h_{\mathrm{res}}=h-h_{\mathrm{ess}},
$$

$\Psi \in C_{c}^{\infty}(0, \infty)^{2}, 0 \leq \Psi \leq 1, \Psi=1$ on an open neighborhood of the point $(\bar{\varrho}, \bar{\vartheta})$

for any measurable function $h$. The idea behind this notation is that it is the essential component $h_{\mathrm{ess}}$ that bears all the relevant information while the residual part $h_{\text {res }}$ disappears in the asymptotic limit, see [21, Chapter 4] for details.

3.1. Coercivity of the relative entropy and uniform bounds. Let $K \subset$ $\bar{K} \subset(0, \infty)^{2}$ be an open set containing $(r, \Theta)$. It follows from relations $(2.4),(2.5)$, and the structural restrictions imposed of the functions $e, s$ in Section 2.3.1 that

$$
\begin{gathered}
H_{\Theta}(\varrho, \vartheta)-\frac{\partial H_{\Theta}(r, \Theta)}{\partial \varrho}(\varrho-r)-H_{\Theta}(r, \Theta) \geq c(K)\left(|\varrho-r|^{2}+|\vartheta-\Theta|^{2}\right) \text { for all }(\varrho, \vartheta) \in K \\
H_{\Theta}(\varrho, \vartheta)-\frac{\partial H_{\Theta}(r, \Theta)}{\partial \varrho}(\varrho-r)-H_{\Theta}(r, \Theta) \\
\geq c(K)(1+\varrho e(\varrho, \vartheta)+\varrho s(\varrho, \vartheta)) \text { whenever }(\varrho, \vartheta) \in[0, \infty)^{2} \backslash K
\end{gathered}
$$

see [21, Chapter 3, Proposition 3.2]. 
3.1.1. First application of the relative entropy inequality. The desired uniform bounds follow immediately from the relative entropy inequality (2.32) evaluated at $r=\bar{\varrho}, \Theta=\bar{\vartheta}, \mathbf{U}=0$ yielding

$$
\begin{aligned}
& {\left[\mathcal{E}_{\varepsilon}\left(\varrho_{\varepsilon}, \vartheta_{\varepsilon}, \mathbf{u}_{\varepsilon} \mid \bar{\varrho}, \bar{\vartheta}, 0\right)\right]_{t=0}^{\tau}} \\
& +\int_{0}^{\tau} \int_{\Omega} \frac{\bar{\vartheta}}{\vartheta}\left(\varepsilon^{a} \mathbb{S}\left(\vartheta_{\varepsilon}, \nabla_{x} \mathbf{u}_{\varepsilon}\right): \nabla_{x} \mathbf{u}_{\varepsilon}-\varepsilon^{b-2} \frac{\mathbf{q}\left(\vartheta_{\varepsilon}, \nabla_{x} \vartheta_{\varepsilon}\right) \cdot \nabla_{x} \vartheta_{\varepsilon}}{\vartheta_{\varepsilon}}\right) \mathrm{d} x \mathrm{~d} t \\
& +\varepsilon^{a-c} \int_{0}^{\tau} \int_{\partial \Omega} \frac{\bar{\vartheta} \beta\left(\vartheta_{\varepsilon}\right)}{\vartheta_{\varepsilon}}\left|\mathbf{u}_{\varepsilon}\right|^{2} \mathrm{~d} \mathrm{~S}_{x} \mathrm{~d} t \leq 0 .
\end{aligned}
$$
we have

Observing that $\mathcal{E}_{\varepsilon}\left(\varrho_{0, \varepsilon}, \vartheta_{0, \varepsilon}, \mathbf{u}_{0, \varepsilon} \mid \bar{\varrho}, \bar{\vartheta}, 0\right)$ remains bounded for $\varepsilon \rightarrow 0$ as soon as

$$
\left\|\varrho_{0, \varepsilon}^{(1)}\right\|_{L^{2} \cap L^{\infty}(\Omega)}+\left\|\vartheta_{0, \varepsilon}^{(1)}\right\|_{L^{2} \cap L^{\infty}(\Omega)}+\left\|\mathbf{u}_{0, \varepsilon}\right\|_{L^{2}\left(\Omega ; R^{3}\right)} \leq c
$$

in (1.14), we deduce the following list of estimates:

$$
\begin{gathered}
\operatorname{ess} \sup _{t \in(0, T)}\left\|\sqrt{\varrho_{\varepsilon}} \mathbf{u}_{\varepsilon}\right\|_{L^{2}\left(\Omega ; R^{3}\right)} \leq c, \\
\operatorname{ess} \sup _{t \in(0, T)}\left\|\left[\frac{\varrho_{\varepsilon}-\bar{\varrho}}{\varepsilon}\right]_{\mathrm{ess}}\right\|_{L^{2}(\Omega)} \leq c, \\
\operatorname{ess} \sup _{t \in(0, T)}\left\|\left[\frac{\vartheta_{\varepsilon}-\bar{\vartheta}}{\varepsilon}\right]_{\mathrm{ess}}\right\|_{L^{2}(\Omega)} \leq c, \\
\operatorname{ess} \sup _{t \in(0, T)}\left[\left\|1_{\mathrm{res}}\right\|_{L^{1}(\Omega)}+\left\|\left[\varrho_{\varepsilon}\right]_{\mathrm{res}}\right\|_{L^{5 / 3}(\Omega)}^{5 / 3}+\left\|\left[\vartheta_{\varepsilon}\right]_{\mathrm{res}}\right\|_{L^{4}(\Omega)}^{4}\right] \leq \varepsilon^{2} c,
\end{gathered}
$$

together with the "integral" bounds:

$$
\begin{gathered}
\varepsilon^{a} \int_{0}^{T} \int_{\Omega}\left|\nabla_{x} \mathbf{u}_{\varepsilon}+\nabla_{x}^{t} \mathbf{u}_{\varepsilon}-\frac{2}{3} \operatorname{div}_{x} \mathbf{u}_{\varepsilon} \mathbb{I}\right|^{2} \mathrm{~d} x \mathrm{~d} t+\varepsilon^{a-c} \int_{0}^{T} \int_{\partial \Omega}\left|\mathbf{u}_{\varepsilon}\right|^{2} \mathrm{~d} \mathrm{~S}_{x} \mathrm{~d} t \leq c \\
\varepsilon^{b-2} \int_{0}^{T} \int_{\Omega}\left|\nabla_{x} \vartheta_{\varepsilon}\right|^{2} \mathrm{~d} x \mathrm{~d} t \leq c
\end{gathered}
$$

where all constants are independent of $\varepsilon \rightarrow 0$.

3.2. Convergence. The uniform bounds $(3.5-3.10)$ are sufficient to pass to the limit in the family of solutions $\left(\varrho_{\varepsilon}, \vartheta_{\varepsilon}, \mathbf{u}_{\varepsilon}\right)$ for $\varepsilon \rightarrow 0$. We obtain, in accordance with (3.5), (3.6), and (3.8),

$$
\begin{aligned}
& \text { ess } \sup _{t \in(0, T)}\left\|\varrho_{\varepsilon}(t, \cdot)-\bar{\varrho}\right\|_{L^{2}(\Omega)+L^{5 / 3}(\Omega)} \leq \varepsilon c \\
& \frac{\vartheta_{\varepsilon}-\bar{\vartheta}}{\varepsilon} \rightarrow \mathcal{T} \text { weakly-(*) in } L^{\infty}\left(0, T ; L^{2}(\Omega)\right),
\end{aligned}
$$

and

$$
\sqrt{\varrho_{\varepsilon}} \mathbf{u}_{\varepsilon} \rightarrow \tilde{\mathbf{u}} \text { weakly- }(*) \text { in } L^{\infty}\left(0, T ; L^{2}\left(\Omega ; R^{3}\right)\right)
$$


3.2.1. Another use of the relative entropy inequality. Of course, our goal is to show that $\tilde{\mathbf{u}}=\sqrt{\bar{\varrho}} \mathbf{v}$, where $\mathbf{v}$ is a solution of the limit Euler system (1.15), (1.16), and that $\mathcal{T}$ solves the transport equation (1.17). To this end, we use again the relative entropy inequality (2.32), this time for the choice of "test functions" that corresponds to the first order $\varepsilon$-approximation. Specifically, we rewrite formally the system $(1.11-1.13)$ as

$$
\begin{gathered}
\varepsilon \partial_{t} \frac{\varrho_{\varepsilon}-\bar{\varrho}}{\varepsilon}+\operatorname{div}_{x}\left(\varrho_{\varepsilon} \mathbf{u}_{\varepsilon}\right)=0 \\
\varepsilon \partial_{t}\left(\varrho_{\varepsilon} \mathbf{u}_{\varepsilon}\right)+\nabla_{x}\left(\partial_{\varrho} p(\bar{\varrho}, \bar{\vartheta}) \frac{\varrho_{\varepsilon}-\bar{\varrho}}{\varepsilon}+\partial_{\vartheta}(\bar{\varrho}, \bar{\vartheta}) \frac{\vartheta_{\varepsilon}-\bar{\vartheta}}{\varepsilon}\right)=\mathbf{F}_{1, \varepsilon}, \\
\partial_{t}\left(\bar{\varrho} \partial_{\vartheta} s(\bar{\varrho}, \bar{\vartheta}) \frac{\vartheta_{\varepsilon}-\bar{\vartheta}}{\varepsilon}+\bar{\varrho} \partial_{\varrho} s(\bar{\varrho}, \bar{\vartheta}) \frac{\varrho_{\varepsilon}-\bar{\varrho}}{\varepsilon}\right) \\
+\operatorname{div}_{x}\left[\left(\bar{\varrho} \partial_{\vartheta} s(\bar{\varrho}, \bar{\vartheta}) \frac{\vartheta_{\varepsilon}-\bar{\vartheta}}{\varepsilon}+\bar{\varrho} \partial_{\varrho} s(\bar{\varrho}, \bar{\vartheta}) \frac{\varrho_{\varepsilon}-\bar{\varrho}}{\varepsilon}\right) \mathbf{u}_{\varepsilon}\right]=F_{2, \varepsilon}
\end{gathered}
$$

where, in view of the uniform bounds established in Section 3.1.1, the "forces" $\mathbf{F}_{1, \varepsilon}$, $F_{2, \varepsilon}$ tend to zero for $\varepsilon \rightarrow 0$.

Thus we have

$$
\varrho_{\varepsilon} \approx \bar{\varrho}+\varepsilon R_{\varepsilon}, \vartheta_{\varepsilon} \approx \bar{\vartheta}+\varepsilon \mathcal{T}_{\varepsilon}, \mathbf{u}_{\varepsilon} \approx \mathbf{v}+\nabla_{x} \Phi_{\varepsilon},
$$

where $\mathbf{v}$ is a solution of the Euler system (1.15), (1.16), and the functions $R_{\varepsilon}, \mathcal{T}_{\varepsilon}, \Phi_{\varepsilon}$ satisfy the acoustic equation

$$
\left\{\begin{array}{c}
\partial_{t}\left(\alpha R_{\varepsilon}+\beta \mathcal{T}_{\varepsilon}\right)+\omega \Delta \Phi_{\varepsilon}=0 \\
\partial_{t} \nabla_{x} \Phi_{\varepsilon}+\nabla_{x}\left(\alpha R_{\varepsilon}+\beta \mathcal{T}_{\varepsilon}\right)=0 \\
\left.\nabla_{x} \Phi_{\varepsilon} \cdot \mathbf{n}\right|_{\partial \Omega}=0
\end{array}\right\}
$$

together with the transport equation

$$
\partial_{t}\left(\delta \mathcal{T}_{\varepsilon}-\beta R_{\varepsilon}\right)+\operatorname{div}_{x}\left[\left(\delta \mathcal{T}_{\varepsilon}-\beta R_{\varepsilon}\right)\left(\mathbf{v}+\nabla_{x} \Phi_{\varepsilon}\right)\right]=0,
$$

see [22] for details.

In accordance with $(3.14-3.16)$,

$$
\alpha=\frac{1}{\varrho} \partial_{\varrho} p(\bar{\varrho}, \bar{\vartheta})>0, \beta=\frac{1}{\bar{\varrho}} \partial_{\vartheta} p(\bar{\varrho}, \bar{\vartheta}), \delta=\bar{\varrho} \partial_{\vartheta} s(\bar{\varrho}, \bar{\vartheta})>0, \omega=\bar{\varrho}\left(\alpha+\frac{\beta^{2}}{\delta}\right)>0 .
$$

The initial values are determined in accordance with (1.14), more specifically, we set

$$
R_{\varepsilon}(0, \cdot)=R_{0, \varepsilon, \delta}=\left[\varrho_{0, \varepsilon}^{(1)}\right]_{\delta}, \mathcal{T}_{\varepsilon}(0, \cdot)=\mathcal{T}_{0, \varepsilon, \delta}=\left[\vartheta_{0, \varepsilon}^{(1)}\right]_{\delta},
$$

while

$$
\mathbf{v}_{0}=\mathbf{H}\left[\mathbf{u}_{0}\right], \quad \nabla_{x} \Phi_{0, \varepsilon}=\nabla_{x} \Phi_{0, \varepsilon \delta}=\left[\mathbf{H}^{\perp}\left[\mathbf{u}_{0, \varepsilon}\right]\right]_{\delta}
$$


where $\mathbf{H}$ denotes the standard Helmholtz projection onto the space of solenoidal functions in $\Omega$, and where $[\cdot]_{\delta}$ are suitable regularizing operators specified in Section 4.2 below. The reason for regularizing the data is that we want to take

$$
r=r_{\varepsilon}=R_{\varepsilon}, \Theta=\Theta_{\varepsilon}=\mathcal{T}_{\varepsilon}, \mathbf{U}=\mathbf{U}_{\varepsilon}=\mathbf{v}+\nabla_{x} \Phi_{\varepsilon}
$$

as test functions in the relative entropy inequality (2.32).

Seeing that

$$
\begin{aligned}
\mathcal{E}_{\varepsilon}\left(\varrho_{\varepsilon}, \vartheta_{\varepsilon}, \mathbf{u}_{\varepsilon} \mid r_{\varepsilon}, \Theta_{\varepsilon}, \mathbf{U}_{\varepsilon}\right)(0) \approx & \int_{\Omega} \varrho_{0, \varepsilon}\left|\mathbf{H}\left[\mathbf{u}_{0, \varepsilon}-\mathbf{u}_{0}\right]+\mathbf{H}^{\perp}\left[\mathbf{u}_{0, \varepsilon}\right]-\left[\mathbf{H}^{\perp}\left[\mathbf{u}_{0, \varepsilon}\right]\right]_{\delta}\right|^{2} \mathrm{~d} x \\
& +\int_{\Omega}\left(\left|\varrho_{0, \varepsilon}^{(1)}-\left[\varrho_{0, \varepsilon}^{(1)}\right]_{\delta}\right|^{2}+\left|\vartheta_{0, \varepsilon}^{(1)}-\left[\vartheta_{0, \varepsilon}^{(1)}\right]_{\delta}\right|^{2}\right) \mathrm{d} x
\end{aligned}
$$

we suppose that

$$
\begin{aligned}
& \varrho_{0, \varepsilon}^{(1)} \rightarrow \varrho_{0}^{(1)} \text { in } L^{2}(\Omega) \text { and weakly-(*) in } L^{\infty}(\Omega), \\
& \vartheta_{0, \varepsilon}^{(1)} \rightarrow \vartheta_{0}^{(1)} \text { in } L^{2}(\Omega) \text { and weakly-(*) in } L^{\infty}(\Omega),
\end{aligned}
$$

and

$$
\mathbf{u}_{0, \varepsilon} \rightarrow \mathbf{u}_{0} \text { in } L^{2}\left(\Omega ; R^{3}\right) .
$$

The leading idea of the proof of convergence towards the limit (target) system is to let first $\varepsilon \rightarrow 0$, then $\delta \rightarrow 0$, in the relative entropy inequality and to use a Gronwall type argument to "absorb" all terms in the remainder on the right-hand side of (2.32). This step was performed in full detail in [22] in the case $\Omega=R^{3}$. The same procedure can be repeated for a general unbounded domain $\Omega \subset R^{3}$ as soon as we make sure that:

- the Euler system (1.15), (1.16) possesses a regular solution on some time interval $\left[0, T_{\max }\right)$ for the initial datum

$$
\mathbf{v}(0, \cdot)=\mathbf{H}\left[\mathbf{u}_{0}\right]
$$

- the acoustic waves described by the system (3.17) become "negligible", meaning vanish, in the asymptotic limit $\varepsilon \rightarrow 0$.

These issues will be addressed in the remaining part of the paper.

3.3. Solvability of the Euler system. The Euler system (1.15), (1.16) is well known to possess local-in-time regular solutions provided the initial datum $\mathbf{v}_{0}$ is sufficiently smooth. Results of this type were obtained by many authors, starting with the pioneering papers by Lichtenstein [40] and Wolibner [52], for more recent results see Beirao da Veiga [5], Kato [31], Kato and Lai [33], among others. Moreover, in their remarkable work, Beale, Kato, and Majda [4] identified a celebrated regularity criterion, namely, the local smooth solution $\mathbf{v}$ can be extended up to the critical time $T_{\max }$ provided

$$
\int_{0}^{T_{\max }}\|\operatorname{curl} \mathbf{v}\|_{L^{\infty}} \mathrm{d} t<\infty .
$$

Of course, these results depend also on the geometry of the underlying physical space $\Omega$. Starting with the known local existence result of Kato and Lai [33] on 
bounded domains, we can construct local-in-time solutions on a general (unbounded) domain $\Omega$ by taking

$$
\Omega_{R}=\Omega \cap\{|x|<R\}
$$

and passing to the limit for $R \rightarrow \infty$. Such a method works provided

- we restrict ourselves to finite energy solutions decaying to zero for $|x| \rightarrow \infty$ in sufficiently high-order Sobolev spaces;

- we are interested only in local-in-time solutions.

Indeed the technique of Kato [31], Kato and Lai [33] is based on energy estimates obtain via multiplication of the equations by $\mathbf{v}$ and its derivatives and the resulting existence time can be taken independent of the size of the domain.

Of course, the situation becomes more delicate if a specific piece of information on the decay rate and/or asymptotic behavior of solutions for $|x| \rightarrow \infty$ is required. The weighted Sobolev space setting to attack this problem was used by Kikuchi [35], Jellouli [28].

In what follows, we shall therefore assume that the initial velocity satisfies

$$
\mathbf{v}_{0}=\mathbf{H}\left[\mathbf{u}_{0}\right] \in W^{k, 2}\left(\Omega ; R^{3}\right) \text { for a certain } k>\frac{5}{2},
$$

for which the Euler system (1.15), (1.16), supplemented with the boundary condition

$$
\left.\mathbf{v} \cdot \mathbf{n}\right|_{\partial \Omega}=0
$$

admits a unique solution on the time interval $\left[0, T_{\max }\right)$ belonging to the class

$$
\mathbf{v} \in C\left(\left[0, T_{\max }\right), W^{k, 2}\left(\Omega, R^{3}\right)\right), \partial_{t} \mathbf{v} \in C\left(\left[0, T_{\max }\right) ; W^{k-1,2}\left(\Omega ; R^{3}\right)\right) .
$$

Note that global existence of solutions to the Euler system, say in the class of weak solutions, is a delicate problem, where many surprising new facts emerged only recently in the work by DeLellis and Székelyhidi [15], [16], Wiedemann [51]. For earlier related results, see Delort [17], the survey by Shnirelman [46] as well as the references cited therein.

4. Acoustic waves. We study the decay properties of solutions to the acoustic equation (3.17) that can be written in a more concise form as

$$
\begin{gathered}
\varepsilon \partial_{t} Z+\Delta \Phi=0, \varepsilon \partial_{t} \Phi+Z=0, \\
\left.\nabla_{x} \Phi \cdot \mathbf{n}\right|_{\partial \Omega}=0, \Phi, Z \rightarrow 0 \text { as }|x| \rightarrow \infty, \\
\Phi(0, \cdot)=\Phi_{0}, Z(0, \cdot)=Z_{0},
\end{gathered}
$$

which is nothing other than a (scaled) linear wave equation for the acoustic potential $\Phi$ supplemented with the homogeneous Neumann boundary conditions. For the sake of simplicity, we dropped the subscript $\varepsilon$ and set $\omega \equiv 1$.

4.1. Neumann Laplacean, Duhamel's formula. The Neumann Laplacean $-\Delta_{N}$ can be viewed as a non-negative self-adjoint operator in the Hilbert space $L^{2}(\Omega)$ with a domain of definition

$$
\begin{aligned}
\mathcal{D}\left(-\Delta_{N}\right)=\left\{w \in W^{1,2}(\Omega) \mid\right. & \int_{\Omega} \nabla_{x} w \cdot \nabla_{x} \phi \mathrm{d} x \\
& \left.=\int_{\Omega} g \phi \mathrm{d} x \text { for a certain } g \in L^{2}(\Omega) \text { and all } \phi \in C_{c}^{\infty}(\bar{\Omega})\right\},
\end{aligned}
$$


where we set

$$
-\Delta_{N} w=g .
$$

As a consequence of the standard elliptic theory, we have

$$
\mathcal{D}\left(-\Delta_{N}\right) \in W_{\text {loc }}^{2,2}(\Omega),
$$

where the estimates can be extended up to the boundary $\partial \Omega$ provided the latter is smooth.

Accordingly, using the standard functional calculus related to $-\Delta_{N}$, we can write solutions of the acoustic equation $(4.1-4.3)$ by means of Duhamel's formula:

$$
\begin{aligned}
\Phi(t, \cdot)= & \frac{1}{2} \exp \left(\mathrm{i} \sqrt{-\Delta_{N}} \frac{t}{\varepsilon}\right)\left[\Phi_{0}-\frac{\mathrm{i}}{\sqrt{-\Delta_{N}}} Z_{0}\right] \\
& +\frac{1}{2} \exp \left(-\mathrm{i} \sqrt{-\Delta_{N}} \frac{t}{\varepsilon}\right)\left[\Phi_{0}+\frac{\mathrm{i}}{\sqrt{-\Delta_{N}}} Z_{0}\right], \\
Z(t, \cdot)= & \frac{1}{2} \exp \left(\mathrm{i} \sqrt{-\Delta_{N}} \frac{t}{\varepsilon}\right)\left[\mathrm{i} \sqrt{-\Delta_{N}}\left[\Phi_{0}\right]+Z_{0}\right] \\
+ & \frac{1}{2} \exp \left(-\mathrm{i} \sqrt{-\Delta_{N}} \frac{t}{\varepsilon}\right)\left[-\mathrm{i} \sqrt{-\Delta_{N}}\left[\Phi_{0}\right]+Z_{0}\right] .
\end{aligned}
$$

4.1.1. Decay and dispersive estimates. Our strategy is based on eliminating the effect of acoustic waves by means of dispersion. In other words, if $\Omega$ is "large", solutions of $(4.1-4.3)$ will decay to zero locally in space as $t \rightarrow \infty$, therefore they will vanish as $\varepsilon \rightarrow 0$ for any positive time. A direct inspection of Duhamel's formula (4.4), (4.5) yields immediately that such a scenario is precluded by the presence of trapped modes - eigenvalues with corresponding eigenfunctions in $L^{2}(\Omega)$. In particular, all bounded domains must be excluded from future analysis.

On the other hand, the existence of eigenvalues of the Neumann Laplacean on a general unbounded domain is a delicate and highly unstable problem, see Davies and Parnovski [14]. Examples of domains, where $\Delta_{N}$ has void point spectrum are $R^{3}$, exterior domains in $R^{3}$, flat waveguides in $R^{3}$, see Lesky and Racke [39].

From now on, we shall therefore assume that the point spectrum of $\Delta_{N}$ defined in $\Omega$ is empty. In such a case, the celebrated RAGE theorem can be used to obtain local decay estimates for solutions of the acoustic equation, see Cycon et al. [12, Theorem $5.8])$ :

TheOREm 4.1. Let $H$ be a Hilbert space, $A: \mathcal{D}(A) \subset H \rightarrow H$ a self-adjoint operator, $C: H \rightarrow H$ a compact operator, and $P_{c}$ the orthogonal projection onto the space of continuity $H_{c}$ of $A$, specifically,

$$
H=H_{c} \oplus \operatorname{cl}_{H}\{\operatorname{span}\{w \in H \mid w \text { an eigenvector of } A\}\} .
$$

Then

$$
\left\|\frac{1}{\tau} \int_{0}^{\tau} \exp (-\mathrm{i} t A) C P_{c} \exp (\mathrm{i} t A) \mathrm{d} t\right\|_{\mathcal{L}(H)} \rightarrow 0 \text { as } \tau \rightarrow \infty
$$


Taking $H=L^{2}(\Omega), A=\sqrt{-\Delta_{N}}, C=\chi^{2} G\left(-\Delta_{N}\right)$, with

$$
\chi \in C_{c}^{\infty}(\bar{\Omega}), \chi \geq 0, G \in C_{c}^{\infty}(0, \infty), 0 \leq G \leq 1,
$$

we may apply Theorem 4.1 for $\tau=1 / \varepsilon$ to obtain

$$
\int_{0}^{T}\left\|\chi G\left(-\Delta_{N}\right) \exp \left(\mathrm{i} \sqrt{-\Delta_{N}} \frac{t}{\varepsilon}\right)[X]\right\|_{L^{2}(\Omega)}^{2} \mathrm{~d} t \leq \omega(\varepsilon)\|X\|_{L^{2}(\Omega)}^{2} \text { for any } X \in L^{2}(\Omega),
$$

where $T>0$ is fixed and

$$
\omega(\varepsilon) \rightarrow 0 \text { as } \varepsilon \rightarrow 0 .
$$

Relation (4.7) is a kind of spatially and "frequency" localized estimates that are quite general and require only the absence of eigenvalues of the operator $\Delta_{N}$ in $\Omega$ and certain smoothness of $\partial \Omega$. The decay rate characterized through $\omega$ may be arbitrarily slow depending on the geometrical properties of $\partial \Omega$, see [20]. The "optimal" rate $\omega(\varepsilon) \approx \varepsilon$ can be achieved provided the operator $\Delta_{N}$ satisfies the limiting absorption principle (LAP), see Leis [38], Vaŭnberg[50] :

We say that $\Delta_{N}$ satisfies the limiting absorption principle (LAP) if the cut-off resolvent operator

$$
\left(1+|x|^{2}\right)^{-s / 2} \circ\left[-\Delta_{N}-\mu \pm \mathrm{i} \delta\right]^{-1} \circ\left(1+|x|^{2}\right)^{-s / 2}, \delta>0, s>1
$$

can be extended as a bounded linear operator on $L^{2}(\Omega)$ for $\delta \rightarrow 0$ and $\mu$ belonging to compact subintervals of $(0, \infty)$.

If $\Delta_{N}$ satisfies (LAP), the relevant alternative to the RAGE theorem is provided by a result of Kato [30] (see also Burq et al. [9]):

Theorem 4.2. [ Reed and Simon [44, Theorem XIII.25 and Corollary] ] Let A be a closed densely defined linear operator and $H$ a self-adjoint densely defined linear operator in a Hilbert space $X$. For $\lambda \notin \mathbb{R}$, let $R_{H}[\lambda]=(H-\lambda \mathrm{Id})^{-1}$ denote the resolvent of $H$. Suppose that

$$
\Gamma=\sup _{\lambda \notin \mathbb{R}, v \in \mathcal{D}\left(A^{*}\right),\|v\|_{X}=1}\left\|A \circ R_{H}[\lambda] \circ A^{*}[v]\right\|_{X}<\infty .
$$

Then

$$
\sup _{w \in X,\|w\|_{X}=1} \frac{\pi}{2} \int_{-\infty}^{\infty}\|A \exp (-\mathrm{i} t H)[w]\|_{X}^{2} \mathrm{~d} t \leq \Gamma^{2}
$$

If $\Delta_{N}$ satisfies (LAP), Theorem 4.2 yields (see [24] for details) the decay rate

$$
\int_{0}^{\infty}\left\|\chi G\left(-\Delta_{N}\right) \exp \left( \pm \mathrm{i} \sqrt{-\Delta_{N}} t\right)[X]\right\|_{L^{2}(\Omega)}^{2} \mathrm{~d} t \leq c\|X\|_{L^{2}(\Omega)}^{2} \text { for any } X \in L^{2}(\Omega),
$$

which is, in fact, equivalent to (4.7) with $\omega(\varepsilon)=\varepsilon$. 
4.2. Smoothing operators. Keeping in mind the solution formulas (4.4), (4.5), we introduce the smoothing operators

$$
\left\{\begin{array}{c}
{[w]_{\delta}=G_{\delta}\left(\sqrt{-\Delta_{N}}\right)[w], G_{\delta} \in C_{c}^{\infty}(R \backslash\{0\}), G_{\delta}(-Z)=G_{\delta}(Z), Z \in R} \\
0 \leq G_{\delta} \leq 1, G_{\delta}(Z) \nearrow 1 \text { as } \delta \rightarrow 0 .
\end{array}\right\}
$$

First, as a consequence of the elliptic regularity,

$$
\left\|[w]_{\delta}\right\|_{W^{k, 2}(\Omega)} \leq c(k, \delta)\|w\|_{L^{2}(\Omega)} \text { for any } k=0,1, \ldots
$$

as long as $\partial \Omega$ is smooth.

Next, we show that $[w]_{\delta}$ decays very fast as $|x| \rightarrow \infty$ for compactly supported $w$. Let us take

$$
\varphi \in C_{c}^{\infty}(\Omega), \operatorname{supp}[\varphi] \subset\{|x|<R\} .
$$

Our goal is to estimate $[\varphi]_{\delta}$ outside the ball $\{|x|<2 R\}$. To this end, we introduce a weighted (pseudo-norm)

$$
\|v\|_{s,(2 R)^{c}}^{2}=\int_{\Omega \cap\{|x|>2 R\}}|v|^{2}|x|^{2 s} \mathrm{~d} x,
$$

and write

$$
G_{\delta}\left(\sqrt{-\Delta_{N}}\right)[\varphi]=\frac{1}{2} \int_{-\infty}^{\infty} \tilde{G}_{\delta}(t)\left(\exp \left(\mathrm{i} \sqrt{-\Delta_{N}} t\right)+\exp \left(-\mathrm{i} \sqrt{-\Delta_{N}} t\right)\right)[\varphi] \mathrm{d} t
$$

where $\tilde{G}_{\delta}$ denotes the Fourier transform of $G_{\delta}$.

Next, we compute

$\left\|G_{\delta}\left(\sqrt{-\Delta_{N}}\right)[\varphi]\right\|_{s,(2 R)^{c}} \leq \frac{1}{2} \int_{-\infty}^{\infty}\left|\tilde{G}_{\delta}(t)\right|\left\|\left(\exp \left(\mathrm{i} \sqrt{-\Delta_{N}} t\right)+\exp \left(-\mathrm{i} \sqrt{-\Delta_{N}} t\right)\right)[\varphi]\right\|_{s,(2 R)^{c}} \mathrm{~d} t$,

where

$$
\begin{aligned}
& \left\|\left(\exp \left(\mathrm{i} \sqrt{-\Delta_{N}} t\right)+\exp \left(-\mathrm{i} \sqrt{-\Delta_{N}} t\right)\right)[\varphi]\right\|_{s,(2 R)^{c}}^{2} \\
& =\int_{\Omega} \operatorname{sgn}^{+}(|x|-2 R)|x|^{2 s}\left|\left(\exp \left(\mathrm{i} \sqrt{-\Delta_{N}} t\right)+\exp \left(-\mathrm{i} \sqrt{-\Delta_{N}} t\right)\right)[\varphi]\right|^{2} \mathrm{~d} x .
\end{aligned}
$$

However, because of the finite speed of propagation of the wave operator $\exp \left( \pm \mathrm{i} \sqrt{-\Delta_{N}} t\right)$, we may infer that

$$
\begin{aligned}
& \int_{\Omega} \operatorname{sgn}^{+}(|x|-2 R)|x|^{2 s}\left|\left(\exp \left(\mathrm{i} \sqrt{-\Delta_{N}} t\right)+\exp \left(-\mathrm{i} \sqrt{-\Delta_{N}} t\right)\right)[\varphi]\right|^{2} \mathrm{~d} x \\
\leq & \operatorname{sgn}^{+}(|t|-R)(|t|+R)^{2 s} \int_{\Omega}\left|\left(\exp \left(\mathrm{i} \sqrt{-\Delta_{N}} t\right)+\exp \left(-\mathrm{i} \sqrt{-\Delta_{N}} t\right)\right)[\varphi]\right|^{2} \mathrm{~d} x \\
\leq & c(s)|t|^{2 s}\|\varphi\|_{L^{2}(\Omega)} ;
\end{aligned}
$$

whence

$$
\left\|G_{\delta}\left(\sqrt{-\Delta_{N}}\right)[\varphi]\right\|_{s,(2 R)^{c}}^{2} \leq c(s, \delta)\|\varphi\|_{L^{2}(\Omega)} \text { provided } \operatorname{supp}[\varphi] \subset\{|x|<r\} .
$$

Applying the same argument to $-\Delta^{\alpha}[\varphi]$ we deduce that $\sup _{x \in \Omega,|x|>2 R}|x|^{s}\left|\partial_{x}^{k}[w]_{\delta}\right| \leq c(s, \delta, k)\|w\|_{L^{2}(\Omega)}$ for all $w \in L^{2}(\Omega), \operatorname{supp}[w] \subset\{|x|<R\}$. 
4.3. Dispersive estimates revisited. The local dispersive estimates (4.7), (4.10) are not strong enough to be used in the analysis of the inviscid limits. Some "global" version is needed, where the cut-off function can be taken $\chi \equiv 1$. Of course, this is not possible with the $L^{2}$-norm as the total energy of acoustic waves is conserved. On the other hand, if $\Omega=R^{3}$, solutions of the system (4.1 - 4.3) satisfy the Strichartz estimates:

$$
\int_{-\infty}^{\infty}\|\exp ( \pm \mathrm{i} \sqrt{-\Delta} t)[h]\|_{L^{q}\left(R^{3}\right)}^{p} \mathrm{~d} t \leq\|h\|_{H^{1,2}\left(R^{3}\right)}^{p}, \frac{1}{2}=\frac{1}{p}+\frac{3}{q}, q<\infty,
$$

where $H^{1,2}$ denotes the homogeneous Sobolev space of functions having first derivatives square integrable in $R^{3}$, see Keel and Tao [34], Strichartz [48].

In addition, the free Laplacean satisfies also the local energy decay in the form

$$
\int_{-\infty}^{\infty}\|\chi \exp ( \pm \mathrm{i} \sqrt{-\Delta} t)[h]\|_{H^{\alpha, 2}\left(R^{3}\right)}^{2} \mathrm{~d} t \leq c(\chi)\|h\|_{H^{\alpha, 2}\left(R^{3}\right)}^{2}, \quad \alpha \leq \frac{3}{2}, \chi \in C_{c}^{\infty}\left(R^{3}\right)
$$

see Smith and Sogge [47, Lemma 2.2].

The estimates (4.14), (4.15) remain valid for the Neumann Laplacean on a "flat" space, for instance, on half-spaces in $R^{3}$, where the functions can be extended as even (with respect to the normal direction) on the whole space $R^{3}$.

4.3.1. Frequency localized Strichartz estimates. We assume that $\Omega$ is a "compact" perturbation of a larger domain on which the Neumann Laplacean satisfies the estimates (4.14), (4.15). For the sake of simplicity, we take the exterior domain $\Omega=R^{3} \backslash K$, where $K$ is a compact, not necessarily connected set. Applications to other domains like local perturbations of a half-space can be handled in a similar manner.

Our goal is to show

$$
\int_{-\infty}^{\infty}\left\|G\left(-\Delta_{N}\right) \exp \left( \pm \mathrm{i} \sqrt{-\Delta_{N}} t\right)[h]\right\|_{L^{q}(\Omega)}^{p} \leq c(G)\|h\|_{H^{1,2}(\Omega)}^{p}, \frac{1}{2}=\frac{1}{p}+\frac{3}{q}, q<\infty
$$

for any $G \in C_{c}^{\infty}(0, \infty)$, adapting the method developed by Burq [8], Smith and Sogge $[47]$.

We start by writing

$$
U(t, \cdot)=G\left(-\Delta_{N}\right) \exp \left( \pm \mathrm{i} \sqrt{-\Delta_{N}} t\right)[h]=\exp \left( \pm \mathrm{i} \sqrt{-\Delta_{N}} t\right) G\left(-\Delta_{N}\right)[h]
$$

as

$$
U=v+w, v=\chi U, w=(1-\chi) U
$$

where

$$
\chi \in C_{c}^{\infty}\left(R^{3}\right), 0 \leq \chi \leq 1, \chi \text { radially symmetric, } \chi(x)=1 \text { for }|x| \leq R,
$$

where $R$ is so large that the ball $\{|x|<R\}$ contains $K$.

Accordingly,

$$
w=w^{1}+w^{2}
$$

where $w^{1}$ solves the homogeneous free wave equation

$$
\partial_{t, t}^{2} w^{1}-\Delta w^{1}=0 \text { in } R^{3},
$$


supplemented with the initial conditions

$$
w^{1}(0)=(1-\chi) G\left(-\Delta_{N}\right)[h], \partial_{t} w^{1}(0)= \pm \mathrm{i}(1-\chi) \sqrt{-\Delta_{N}} G\left(-\Delta_{N}\right)[h],
$$

while

$$
\begin{gathered}
\partial_{t, t}^{2} w^{2}-\Delta w^{2}=F \text { in } R^{3}, \\
w^{2}(0)=\partial_{t} w^{2}(0)=0,
\end{gathered}
$$

with

$$
F=-\nabla_{x} \chi \nabla_{x} U-U \Delta \chi .
$$

As a consequence of the standard Strichartz estimates (4.14), we get

$$
\int_{-\infty}^{\infty}\left\|w^{1}\right\|_{L^{q}\left(R^{3}\right)}^{p} \mathrm{~d} t \leq c(G)\|h\|_{H^{1,2}\left(R^{3}\right)}^{p}, \frac{1}{2}=\frac{1}{p}+\frac{3}{q}, q<\infty .
$$

Furthermore, using Duhamel's formula, we obtain

$$
\begin{aligned}
w^{2}(\tau, \cdot)= & \frac{1}{2 \sqrt{-\Delta}}\left[\exp (\mathrm{i} \sqrt{-\Delta} \tau) \int_{0}^{\tau} \exp (-\mathrm{i} \sqrt{-\Delta} s)\left[\eta^{2} F(s)\right] \mathrm{d} s\right] \\
& -\frac{1}{2 \sqrt{-\Delta}}\left[\exp (-\mathrm{i} \sqrt{-\Delta} \tau) \int_{0}^{\tau} \exp (\mathrm{i} \sqrt{-\Delta} s)\left[\eta^{2} F(s)\right] \mathrm{d} s\right],
\end{aligned}
$$

with

$$
\eta \in C_{c}^{\infty}\left(R^{3}\right), 0 \leq \eta \leq 1, \eta \text { radially symmetric, } \eta=1 \text { on } \operatorname{supp}[F] .
$$

Similarly to [8], we use the following result of of Christ and Kiselev [11]:

Lemma 4.1. Let $X$ and $Y$ be Banach spaces and assume that $K(t, s)$ is a continuous function taking its values in the space of bounded linear operators from $X$ to Y. Set

$$
\mathcal{T}[f](t)=\int_{a}^{b} K(t, s) f(s) \mathrm{d} s, \mathcal{W}[f](t)=\int_{a}^{t} K(t, s) f(s) \mathrm{d} s
$$

where

$$
0 \leq a \leq b \leq \infty
$$

Suppose that

$$
\|\mathcal{T}[f]\|_{L^{p}(a, b ; Y)} \leq c_{1}\|f\|_{L^{r}(a, b ; X)}
$$

for certain

$$
1 \leq r<p \leq \infty
$$

Then

$$
\|\mathcal{W}[f]\|_{L^{p}(a, b ; Y)} \leq c_{2}\|f\|_{L^{r}(a, b ; X)},
$$


where $c_{2}$ depends only on $c_{1}, p$, and $r$.

We aim to apply Lemma 4.1 in the situation

$$
X=L^{2}\left(R^{3}\right), Y=L^{q}\left(R^{3}\right), q<\infty, \frac{1}{2}=\frac{1}{p}+\frac{3}{q}, r=2,
$$

and

$$
f=F, K(t, s)[F]=\frac{1}{\sqrt{-\Delta}} \exp ( \pm \mathrm{i} \sqrt{-\Delta}(t-s))\left[\eta^{2} F\right] .
$$

Writing

$$
\int_{0}^{\infty} K(t, s) F(s) \mathrm{d} s=\exp ( \pm \mathrm{i} \sqrt{-\Delta} t) \frac{1}{\sqrt{-\Delta}} \int_{0}^{\infty} \exp (\mp \mathrm{i} \sqrt{-\Delta} s)\left[\chi^{2} F(s)\right] \mathrm{d} s
$$

we have to show, keeping in mind the Strichartz estimates (4.14), that

$$
\left\|\int_{0}^{\infty} \exp ( \pm \mathrm{i} \sqrt{-\Delta} s)\left[\eta^{2} F(s)\right] \mathrm{d} s\right\|_{L^{2}\left(R^{3}\right)} \leq c\|F\|_{L^{2}\left(0, \infty ; L^{2}\left(R^{3}\right)\right)} .
$$

However,

$$
\begin{aligned}
& \left\|\int_{0}^{\infty} \exp ( \pm \mathrm{i} \sqrt{-\Delta} s)\left[\chi^{2} F(s)\right] \mathrm{d} s\right\|_{L^{2}\left(R^{3}\right)} \\
= & \sup _{\|v\|_{L^{2}\left(R^{3}\right)} \leq 1} \int_{0}^{\infty}\left\langle\exp ( \pm \mathrm{i} \sqrt{-\Delta} s)\left[\chi^{2} F(s)\right] ; v\right\rangle \mathrm{d} s \\
= & \sup _{\|v\|_{L^{2}\left(R^{3}\right)} \leq 1} \int_{0}^{\infty}\langle\chi F(s) ; \chi \exp (-\mathrm{i} \sqrt{-\Delta} s)[v]\rangle \mathrm{d} s ;
\end{aligned}
$$

whence the desired conclusion (4.18) follows from the local energy decay estimates stated in (4.15).

As the norm of $F$ is bounded in view of the local estimates established in (4.15), we may infer that

$$
\int_{-\infty}^{\infty}\left\|w^{2}\right\|_{L^{q}\left(R^{3}\right)}^{p} \mathrm{~d} t \leq c(G)\|h\|_{H^{1,2}\left(R^{3}\right)}^{p}, \frac{1}{2}=\frac{1}{p}+\frac{3}{q}, q<\infty .
$$

Finally, since $v=\chi U$ is compactly supported, we deduce from (4.15) combined with the standard elliptic regularity theory of $\Delta_{N}$ that

$$
\int_{0}^{\infty}\|v\|_{L^{q}(\Omega)}^{2} \mathrm{~d} t \leq c(G)\|h\|_{H^{1,2}(\Omega)}^{2}
$$

while, by virtue of the energy estimates,

$$
\sup _{t>0}\|v(t, \cdot)\|_{L^{q}(\Omega)} \leq c(G)\|h\|_{H^{1,2}(\Omega)},
$$

where $q<\infty$ is the same as in (4.16).

Interpolating (4.20), (4.21) and combining the result with the previous estimates, we get (4.16). As a matter of fact, our conclusion can be "strengthened" to: 


$$
\int_{-\infty}^{\infty}\left\|G\left(-\Delta_{N}\right) \exp \left( \pm \mathrm{i} \sqrt{-\Delta_{N}} t\right)[h]\right\|_{L^{q}(\Omega)}^{p} \leq c(G)\|h\|_{L^{2}(\Omega)}^{p}, \frac{1}{2}=\frac{1}{p}+\frac{3}{q}, q<\infty
$$

for any $G \in C_{c}^{\infty}(0, \infty)$.

In accordance with the previous discussion, we say that a domain $\Omega \subset R^{3}$ is admissible if:

- $\Omega$ is an (unbounded) smooth domain in $R^{3}$, on which the Neumann Laplacean $\Delta_{N}$ satisfies the limiting absorption principle (4.8).

- There is $R>0$ and a domain $D \subset R^{3}$ such that $\Delta_{N}$ satisfies the Strichartz and local decay estimates (4.14), (4.15) on $D$ and $D \cap\{|x|>R\}=\Omega \cap\{|x|>$ $R\}$.

As we have just observed, the Neumann Laplacean $\Delta_{N}$ satisfies the frequency localized Strichartz estimates (4.22) as soon as $\Omega$ is an admissible domain. Typically, the "reference" domain $D$ is taken $R^{3}$ or a half-space in applications.

5. Conclusion. The uniform estimates established in Section 4, specifically (4.22), are sufficient to pass to the limit in the relative entropy inequality, first $\varepsilon \rightarrow 0$, then $\delta \rightarrow 0$. This step can be performed exactly as in [22]. Thus we have shown (strong) convergence of the dissipative solutions of the scaled Navier-Stokes-Fourier system $(1.11$ - 1.14) to the (unique) solution of the target problem $(1.15-1.17)$, endowed with the initial data

$$
\mathbf{v}_{0}=\mathbf{H}\left[\mathbf{u}_{0}\right], \mathcal{T}(0, \cdot)=\bar{\varrho} \partial_{\vartheta} s(\bar{\varrho}, \bar{\vartheta}) \vartheta_{0}^{(1)}-\frac{1}{\bar{\varrho}} \partial_{\vartheta} p(\bar{\varrho}, \bar{\vartheta}) \varrho_{0}^{(1)} .
$$

The convergence takes place on any compact time interval $\left[T_{1}, T_{2}\right]$ provided $0<T_{1}<$ $T_{2}<T_{\max }$, where $T_{\max } \leq \infty$ is the life span of the smooth solution to the target system. The details of the proof can be found in [22].

Let us summarize our results that may be viewed as a generalization of $[22$, Theorem 3.1] to the class of admissible domains introduced in Section 4.3.1:

THEOREM 5.1. Let $\Omega \subset R^{3}$ be an admissible domain in the sense specified in Section 4.3.1. Suppose that the thermodynamic functions $p, e, s$ and the transport coefficients $\mu, \lambda, \kappa$, and $\beta$ comply with the structural restrictions introduced in Section 2.3.1, with

$$
b>0,0 \leq c<a<\frac{10}{3},
$$

Furthermore, suppose that the initial data (1.14) satisfy

$$
\left\{\varrho_{0, \varepsilon}^{(1)}\right\}_{\varepsilon>0}, \quad\left\{\vartheta_{0, \varepsilon}^{(1)}\right\}_{\varepsilon>0} \text { bounded in } L^{2} \cap L^{\infty}(\Omega), \varrho_{0, \varepsilon}^{(1)} \rightarrow \varrho_{0}^{(1)}, \vartheta_{0, \varepsilon}^{(1)} \rightarrow \vartheta_{0}^{(1)} \text { in } L^{2}(\Omega),
$$

and

$$
\mathbf{u}_{0, \varepsilon} \rightarrow \mathbf{u}_{0} \text { in } L^{2}\left(\Omega ; R^{3}\right),
$$

where

$$
\varrho_{0}^{(1)}, \vartheta_{0}^{(1)} \in W^{1,2} \cap W^{1, \infty}(\Omega), \mathbf{H}\left[\mathbf{u}_{0}\right]=\mathbf{v}_{0} \in W^{k, 2}\left(\Omega ; R^{3}\right) \text { for a certain } k>\frac{5}{2} .
$$


Let $T_{\max } \leq \infty$ be the maximal life-span of the regular solution $\mathbf{v}$ to the Euler system (1.15), (1.16), with the initial datum $\mathbf{v}(0, \cdot)=\mathbf{v}_{0}$. Finally, let $\left\{\varrho_{\varepsilon}, \vartheta_{\varepsilon}, \mathbf{u}_{\varepsilon}\right\}_{\varepsilon>0}$ be a family of dissipative weak solutions of the scaled Navier-Stokes-Fourier system (1.11 - 1.14) in $(0, T) \times R^{3}, T<T_{\max }$, supplemented with the boundary conditions (1.18), (1.19).

Then

$$
\operatorname{ess} \sup _{t \in(0, T)}\left\|\varrho_{\varepsilon}(t, \cdot)-\bar{\varrho}\right\|_{L^{2}+L^{5 / 3}(\Omega)} \leq \varepsilon c
$$

$\sqrt{\varrho_{\varepsilon}} \mathbf{u}_{\varepsilon} \rightarrow \sqrt{\bar{\varrho}} \mathbf{v}$ in $L_{\mathrm{loc}}^{\infty}\left((0, T] ; L_{\mathrm{loc}}^{2}\left(\Omega ; R^{3}\right)\right)$ and weakly-(*) in $L^{\infty}\left(0, T ; L^{2}\left(\Omega ; R^{3}\right)\right)$

and

$\frac{\vartheta_{\varepsilon}-\bar{\vartheta}}{\varepsilon} \rightarrow \mathcal{T}$ in $L_{\mathrm{loc}}^{\infty}\left((0, T] ; L_{\mathrm{loc}}^{q}(\Omega)\right), 1 \leq q<2$, and weakly-( $\left.{ }^{*}\right)$ in $L^{\infty}\left(0, T ; L^{2}(\Omega)\right)$,

where $\mathbf{v}, \mathcal{T}$ is the unique solution of the Euler-Boussinesq system (1.15 - 1.17), with the initial data

$$
\mathbf{v}_{0}=\mathbf{H}\left[\mathbf{u}_{0}\right], \quad \mathcal{T}_{0}=\bar{\varrho} \frac{\partial s(\bar{\varrho}, \bar{\vartheta})}{\partial \vartheta} \vartheta_{0}^{(1)}-\frac{1}{\bar{\varrho}} \frac{\partial p(\bar{\varrho}, \bar{\vartheta})}{\partial \vartheta} \varrho_{0}^{(1)}
$$

5.1. Related results, alternative techniques. An alternative approach to singular limits is based on strong solutions for both the primitive and the target system, see Klainerman and Majda [36]. Necessarily, the results are only local-in-time even if the target system happens to admit a global solution for a specific choice of the data. The initial data for the primitive system must be regular and their convergence to the limit values takes place in stronger topologies. We refer to Alazard [1], [2], [3] for very interesting results concerning the full Navier-Stokes-Fourier system.

There is a vast amount of literature concerning the incompressible limits of both viscous (Navier-Stokes) or inviscid (Euler) systems. The dispersive estimates were used by Danchin [13], Desjardins and Grenier [18], Ukai [49], for more applications of the RAGE theorem in this context, see Métivier and Schochet [43]. The interested reader may salso consult the surveys by Gallagher [26], Masmoudi [42], and Schochet [45] for more material.

\section{REFERENCES}

[1] T. AlazARD, Incompressible limit of the nonisentropic Euler equations with the solid wall boundary conditions, Adv. Differential Equations, 10:1 (2005), pp. 19-44.

[2] T. AlazArD, Low Mach number flows and combustion, SIAM J. Math. Anal., $38: 4$ (2006), pp. 1186-1213 (electronic).

[3] T. Alazard, Low Mach number limit of the full Navier-Stokes equations, Arch. Rational Mech. Anal., 180 (2006), pp. 1-73.

[4] J. T. BeAle, T. KATO, AND A. MAJDA, Remarks on the breakdown of smooth solutions for the 3-D Euler equations, Comm. Math. Phys., 94:1 (1984), pp. 61-66.

[5] H. Beirao DA Veiga, A well posedness theorem for non-homogeneous inviscid fluids via a perturbation theorem, J. Differential Equations, 78 (1989), pp. 308-319.

[6] D. Bresch And B. Desjardins, Stabilité de solutions faibles globales pour les équations de Navier-Stokes compressibles avec température, C.R. Acad. Sci. Paris, 343 (2006), pp. 219224. 
[7] D. Bresch And B. Desjardins, On the existence of global weak solutions to the Navier-Stokes equations for viscous compressible and heat conducting fluids, J. Math. Pures Appl., 87 (2007), pp. 57-90.

[8] N. BuRQ, Global Strichartz estimates for nontrapping geometries: about an article by $H$. F. Smith and C. D. Sogge: "Global Strichartz estimates for nontrapping perturbations of the Laplacian", Comm. Partial Differential Equations, 28 (9-10) (2003), pp. 1675-1683.

[9] N. Burq, F. Planchon, J. G. Stalker, and A. S. Tahvildar-Zadeh, Strichartz estimates for the wave and Schrödinger equations with potentials of critical decay, Indiana Univ. Math. J., 53:6 (2004), pp. 1665-1680.

[10] H. Callen, Thermodynamics and an Introduction to Thermostatistics, Wiley, New Yoerk, 1985.

[11] M. Christ and A. Kiselev, Maximal functions associated to filtrations, J. Funct. Anal., 179:2 (2001), pp. 409-425.

[12] H. L. Cycon, R. G. Froese, W. Kirsch, And B. Simon, Schrödinger operators: with applications to quantum mechanics and global geometry, Texts and monographs in physics, Springer-Verlag, Berlin,Heidelberg, 1987.

[13] R. DANCHIN, Low Mach number limit for viscous compressible flows, M2AN Math. Model Numer. Anal., 39 (2005), pp. 459-475.

[14] E. B. Davies And L. PARnovski, Trapped modes in acoustic waveguides, Quart. J. Mech. Appl. Math., 51:3 (1998), pp. 477-492.

[15] C. De Lellis And L. SzÉKelyhidi, JR, The h-principle and the equations of fluid dynamics, Bull. Amer. Math. Soc. (N.S.), 49L:3 (2012), pp. 347-375.

[16] C. Delellis And L. SzÉKelyhidi, Dissipative Euler flows and Onsager conjecture, 2012, preprint.

[17] J.-M. Delort, Existence de nappes de tourbillon en dimension deux, J. Amer. Math. Soc., 4:3 (1991), pp. 553-586.

[18] B. Desjardins And E. GRenier, Low Mach number limit of viscous compressible flows in the whole space, R. Soc. Lond. Proc. Ser. A Math. Phys. Eng. Sci., 455 (1999), pp. 2271-2279.

[19] J. L. ERICKSEN, Introduction to the thermodynamics of solids, revised ed., Applied Mathematical Sciences, vol. 131, Springer-Verlag, New York, 1998.

[20] E. FEIREISL, Local decay of acoustic waves in the low Mach number limits on general unbounded domains under slip boundary conditions, Commun. Partial Differential Equations, 2010. Submitted.

[21] E. Feireisl And A. Novotný, Singular limits in thermodynamics of viscous fluids, BirkhäuserVerlag, Basel, 2009.

[22] E. FeIREISL AND A. Novotný, Inviscid incompressible limits of the full Navier-Stokes-Fourier system, Commun. Math. Phys., 2012, to appear.

[23] E. FEIREISL AND A. NovotnÝ. Weak-strong uniqueness property for the full Navier-StokesFourier system, Arch. Rational Mech. Anal., 204 (2012), pp. 683-706.

[24] E. Feireisl, A. Novotný, And H. Petzeltová, Low Mach number limit for the Navier-Stokes system on unbounded domains under strong stratification, Commun. Partial Differential Equations, 35 (2010), pp. 68-88.

[25] E. FEIREISL AND D. PRAŽÁK, Asymptotic behavior of dynamical systems in fluid mechanics, AIMS, Springfield, 2010.

[26] I. Gallagher, Résultats récents sur la limite incompressible, Astérisque, (299):Exp. No. 926, vii, pp. 29-57, 2005. Séminaire Bourbaki. Vol. 2003/2004.

[27] G. Gallavotti, Foundations of fluid dynamics, Springer-Verlag, New York, 2002.

[28] M. Jellouli, Équation d'Euler sur un domaine extérieur. Existence locale et apparition de singularités, Osaka J. Math., 38:3 (2001), pp. 619-647.

[29] D. Jesslé, B. J. Jin, AND A. Novotný, Navier-Stokes-Fourier system on unbounded domains: weak solutions, relative entropies, weak-strong uniqueness, 2012. Preprint.

[30] T. KATO, Wave operators and similarity for some non-selfadjoint operators, Math. Ann., 162 (1965/1966), pp. 258-279.

[31] T. KAтo, On classical solutions of the two-dimensional nonstationary Euler equation, Arch. Rational Mech. Anal., 25 (1967), pp. 188-200.

[32] T. KAто, Remarks on the zero viscosity limit for nonstationary Navier-Stokes flows with boundary, in Seminar on PDE's, S.S. Chern (ed.), Springer, New York, 1984.

[33] T. Kato And C. Y. LAI, Nonlinear evolution equations and the Euler flow, J. Funct. Anal., 56 (1984), pp. 15-28.

[34] M. KeEl AND T. TAO, Endpoint Strichartz estimates, Amer. J. Math., 120:5 (1998), pp. 955980.

[35] K. KIKUCHI, The existence and uniqueness of nonstationary ideal incompressible flow in exte- 
rior domains in $\mathbf{R}^{3}$, J. Math. Soc. Japan, 38:4 (1986), pp. 575-598.

[36] S. Klainerman And A. MAJdA, Singular limits of quasilinear hyperbolic systems with large parameters and the incompressible limit of compressible fluids, Comm. Pure Appl. Math., 34 (1981), pp. 481-524.

[37] R. Klein, N. Botta, T. Schneider, C. D. Munz, S. Roller, A. Meister, L. Hoffmann, AND T. SONAR, Asymptotic adaptive methods for multi-scale problems in fluid mechanics, J. Engrg. Math., 39 (2001), pp. 261-343.

[38] R. LEIS, Initial-boundary value problems in mathematical physics, B.G. Teubner, Stuttgart, 1986.

[39] P. H. LESKY AND R. RACKE, Elastic and electro-magnetic waves in infinite waveguides, J. Differential Equations, 244:4 (2008), pp. 945-971.

[40] L. Lichtenstein, Über einige Existenzprobleme der Hydrodynamik, Math. Z., 26:1 (1927), pp 196-323.

[41] P.-L. Lions, Mathematical topics in fluid dynamics, Vol.1, Incompressible models, Oxford Science Publication, Oxford, 1996.

[42] N. Masmoudi, Examples of singular limits in hydrodynamics, in Handbook of Differential Equations, III, C. Dafermos, E. Feireisl Eds., Elsevier, Amsterdam, 2006.

[43] G. MÉtivier And S. Schochet, The incompressible limit of the non-isentropic Euler equations, Arch. Rational Mech. Anal., 158 (2001), pp. 61-90.

[44] M. ReEd And B. Simon, Methods of modern mathematical physics. IV. Analysis of operators, Academic Press [Harcourt Brace Jovanovich Publishers], New York, 1978.

[45] S. Schochet, The mathematical theory of low Mach number flows, M2ANMath. Model Numer. anal., 39 (2005), pp. 441-458.

[46] A. Shnirelman, Weak solutions of incompressible Euler equations, in "Handbook of mathematical fluid dynamics, Vol. II", pages 87-116. North-Holland, Amsterdam, 2003.

[47] H. F. Smith And C. D. SogGe, Global Strichartz estimates for nontrapping perturbations of the Laplacian, Comm. Partial Differential Equations, 25(11-12) (2000), pp. 2171-2183.

[48] R. S. Strichartz, A priori estimates for the wave equation and some applications, J. Functional Analysis, 5 (1970), pp. 218-235.

[49] S. UKAI, The incompressible limit and the initial layer of the compressible Euler equation, J. Math. Kyoto Univ., 26:2 (1986), pp. 323-331.

[50] B. R. VAĬNBERG, Asimptoticheskie metody v uravneniyakh matematicheskoi fiziki, Moskov. Gos. Univ., Moscow, 1982.

[51] E. Wiedemann, Existence of weak solutions for the incompressible Euler equations, Ann. Inst. H. Poincaré Anal. Non Linéaire, 28:5 (2011), pp. 727-730.

[52] W. Wolibner, Un theorème sur l'existence du mouvement plan d'un fluide parfait, homogène, incompressible, pendant un temps infiniment long, Math. Z., 37:1 (1933), pp. 698-726. 
E. FEIREISL 